\title{
A Study of Patterns and Etiologies of Peripheral Lymphadenopathy in a Tertiary Care Hospital in Ranchi,India
}

\author{
Brajesh Mishra $^{1}$, Apporv Sinha ${ }^{2}$, Shashi Bhushan Singh ${ }^{3 *}$ \\ ${ }^{1}$ Assistant Professor, ${ }^{2}$ Senior Resident, Department of TB. \& Chest,,${ }^{3 *}$ Associate Professor cum statistician, \\ PSM Deptt., and corresponding Author, Rajendra Institute of Medical Sciences(RIMS), Ranchi.
}

\begin{abstract}
:
Background- Peripheral lymphadenopathy (LAP) is a common clinical condition and presents a diagnostic dilemma especially when there is no obvious cause. Fine needle aspiration cytology(FNAC) is usually the first step diagnostic procedure. The patterns and etiologies of peripheral lymphadenopathy can vary depending on geographical areas. The present study was undertaken to investigate the patterns of presentation and the various etiologies of peripheral lymphadenopathy in Ranchi, on the basis of cytological \& radiological examination, and correlate the findings.

Methods- This observational descriptive study included 119 subjects of peripheral lymphadenopathy visiting the Deptt. of TB \& Chest, RIMS, Ranchi. All patients were subjected to Fine-needle aspiration cytology $(F N A C)$ and chest $x$-ray (CXR) along with other relevant investigations. Diagnosis for etiology was established on the basis of FNAC findings. Cytological findings were correlated with findings of chest $x$-ray showing Mediastinal involvement, Lung parenchyma involvement and/or Pleural effusion. All data was compiled and presented in tabular and/or graphical form. Results were correlated and compared with other studies.

Results- Males showed a higher incidence than females(66\% vs 34\%). Most cases fell in the 21-30 yr age group (39.4\%). Tuberculous lymphadenitis was the most frequent cytopathological finding(52.1\%) followed by reactive( $21 \%)$, pyogenic(18.5\%), metastatic(5\%) and lymphoma(3.4\%). Malignant findings were seen in older age groups. Cervical lymph nodes were most frequently effected(45.3\%), followed by supraclavicular(22.6\%), axillary(18.4\%), submental(7.5\%), post-auricular(5\%) and inguinal (0.08\%). Tuberculous lesions were more common in cervical whereas malignant changes were also frequent in cervical \& Supraclavicular groups. Abnormal chest X-ray findings were more common in Tuberculous and malignant lesions.

Conclusion-It was found that the majority of patients presenting with LAP were diagnosed having tuberculous lesions most likely due to a high prevalence of TB in Ranchi zone. FNAC is a simple, safe procedure and can be accepted as a reliable technique to establish a cytological diagnosis in lymphadenopathies. Similar pattern was observed in some other geographical areas in India. Further procedure for histopathological study may be required in some cases associated with multiple other risk factors.
\end{abstract}

Keywords- Peripheral Lymphadenopathy, FNAC, Ranchi, Tuberculous Lymphadenitis, LAP

\section{Introduction}

Lymphadenopathy refers to the conditions in which lymph nodes become abnormal in size, consistency, and number. Normal lymph nodes usually measure less than one $\mathrm{cm}$ in diameter. ${ }^{1}$ Enlarged peripheral lymph nodes are common and may be asymptomatic. Peripheral lymphadenopathy(LAP) presents a diagnostic dilemma especially when there is no obvious cause after the history and physical examination. In unexplained cases of LAP where the cause is not obvious on primary evaluation, laboratory tests, tissue examination and radiological studies are recommended for diagnosis. Imaging can identify the size and distribution of the node more accurately than physical examination. Tissue diagnosis by fine needle aspiration cytology(FNAC) or excisional biopsy is the diagnostic procedure of choice for LAP. ${ }^{2}$

The patterns and etiologies of peripheral lymphadenopathy can vary depending on geographical areas. ${ }^{1,3}$ Besides this, age, sex and environmental exposure are important determinants of the etiology of peripheral lyphadenopathy. Studies have emphasized on the importance of geographical and environmental factors on the incidence, pattern and etiologies of LAP. ${ }^{3}$ This is especially because of wide variation in the prevalence of tuberculosis (TB) and cancers in different parts of India ${ }^{\mathbf{4 , 5 , 6}}$. Tobacco, alcoholism and other regional environmental factors also effect the overall clinicopathological presentation of LAP.

Ranchi city has a distinctly unique geographical and socio-economic profile. Rajendra Institute of Medical Sciences (RIMS) is a medical institute of Ranchi University in the capital of Jharkhand, India which provides services to a large number of patients from in and around Ranchi. The present study was undertaken to investigate the patterns of presentation and the various etiologies of peripheral lymphadenopathy in Ranchi, on the basis of cytological \& radiological examination and correlate the findings. 


\section{Methods}

The present observational descriptive study was conducted in Department of TB. \& Chest, Rajendra Institute of Medical Sciences (RIMS) , Ranchi between November 2013 to October 2016. Patients(10 years and above) presenting to the department with palpable lymph nodes were considered for a detailed clinical history. A total of 119 patients were included in the study. All patients with peripheral lymphadenopathy underwent a complete physical examination. Palpable lymph nodes were evaluated for its location, size, fixation, consistency and tenderness. All patients were subjected to Fine-needle aspiration cytology (FNAC) and chest x-ray (CXR) from respective department along with other relevant investigations. Available patient data was classified by age and sex.

Diagnosis for etiology was established on the basis of FNAC findings . Diagnosed cases were furthur sub-classified based on the regional groups of lymph nodes involved. Cytological findings were correlated with findings of chest $\mathrm{x}$-ray showing Mediastinal involvement, Lung parenchyma involvement and/or Pleural effusion. Data was entered in Microsoft Excel(2007). All data was compiled and presented in tabular and/or graphical form, percentages were calculated wherever relevant.

\section{Results}

A total of 119 patients with peripheral lymphadenopathy were included for cytopathological diagnosis and radiological evaluation. Figure -1 shows age and sex wise distribution of the study population. Maximum number of cases ( 25 males and 22 females) were in the 21 to 30 yrs age group. Fewer cases were observed in the older age groups. Males showed a higher prevalence as compared to females(66\% vs $34 \%)$.

Figure-1. Age and sex wise distribution of subjects with peripheral lymphadenopathy.

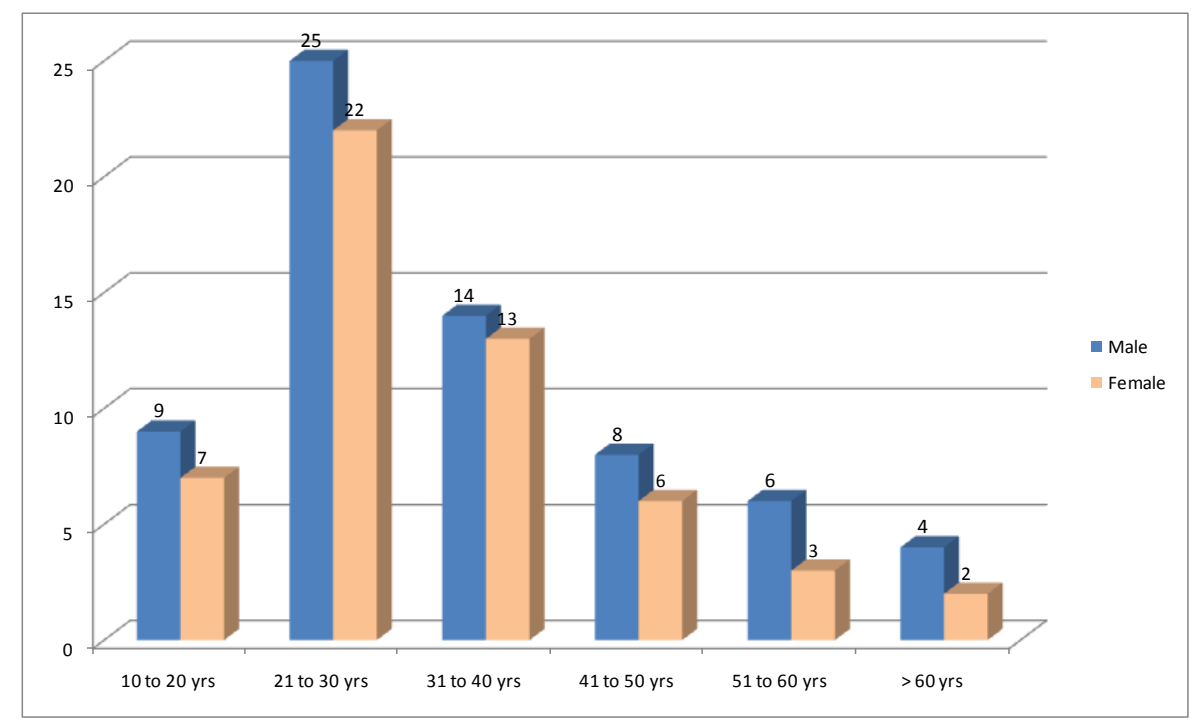

Figure1: Bargraph showing the sex distribution of the patients according to different age group

Results of cytopathological evaluation, Based on Fine Needle Aspiration Cytology are shown in table- 1. Among the common etiologies, Tuberculous lymphadenitis was found to be the most frequent cause of LAP, followed by reactive and pyogenic causes. Lymphoma and metastasis from other sites were found to be least frequent causes.

Table- 1: Cytological diagnosis of patients with peripheral Lymphadenopathy (no=119)

\begin{tabular}{|l|l|l|}
\hline Cytological Diagnosis & No of cases & Percentage (\%) \\
\hline Tuberculous lympadinitis & 62 & $52.1 \%$ \\
\hline Reactive lymphadenitis & 25 & $21.0 \%$ \\
\hline Pyogenic Lymphadinitis & 22 & $18.5 \%$ \\
\hline Lymphoma & 4 & $3.4 \%$ \\
\hline Metastatic lymphadenopathy & 6 & $5.0 \%$ \\
\hline & 119 & $100 \%$ \\
\hline
\end{tabular}

Diagnostic data collected was re-tabulated on the basis of age and sex distribution of different etiologies (Table-2). It was observed that the most frequent cause in youngest age group(10-20 yrs) was Reactive lymphadenopathy. As is apparent from table-2, Tuberculous lymphadenitis was the most common etiology in the young-to-middle age population(between 21 to $60 \mathrm{yrs}$ ). In the older population (> $60 \mathrm{yrs}$ ),

DOI: 10.9790/0853-1605074650 www.iosrjournals.org $\quad 47$ |Page


Metastatic lymphadenopathy was most common. Relatively higher number of cases of Lymphoma and pyogenic lymphadenitis were seen in the 21-30 yrs age group.

Table 2 : Age and sex wise distribution of peripheral lymphadenopathy

\begin{tabular}{|c|c|c|c|c|c|c|c|c|c|c|c|c|}
\hline \multirow{3}{*}{$\begin{array}{l}\text { Cytological } \\
\text { diagnosis }\end{array}$} & \multicolumn{12}{|c|}{ Age (yrs) and sex distribution } \\
\hline & \multicolumn{2}{|c|}{$10-20$} & \multicolumn{2}{|c|}{$21-30$} & \multicolumn{2}{|c|}{$31-40$} & \multicolumn{2}{|c|}{$41-50$} & \multicolumn{2}{|c|}{$51-60$} & \multicolumn{2}{|c|}{$>60$} \\
\hline & $\mathrm{M}$ & $\mathrm{F}$ & $\mathrm{M}$ & F & $\mathrm{M}$ & $\bar{F}$ & $\mathrm{M}$ & F & $\mathrm{M}$ & $\mathrm{F}$ & $\mathrm{M}$ & $\mathrm{F}$ \\
\hline Tuberculous & 2 & 2 & 13 & 11 & 10 & 8 & 4 & 4 & 4 & 2 & 1 & 1 \\
\hline Reactive & 5 & 3 & 2 & 6 & 3 & 3 & 1 & 2 & 0 & 0 & 0 & 0 \\
\hline Pyogenic & 2 & 1 & 8 & 5 & 1 & 2 & 2 & 0 & 1 & 0 & 0 & 0 \\
\hline Lymphoma & 0 & 1 & 2 & 0 & 0 & 0 & 0 & 0 & 1 & 0 & 0 & 0 \\
\hline \multirow[t]{3}{*}{ metastatic } & 0 & 0 & 0 & 0 & 0 & 0 & 1 & 0 & 0 & 1 & 3 & 1 \\
\hline & 9 & 7 & 25 & 22 & 14 & 13 & 8 & 6 & 6 & 3 & 4 & 2 \\
\hline & \multicolumn{2}{|l|}{16} & \multicolumn{2}{|l|}{47} & \multicolumn{2}{|l|}{27} & \multicolumn{2}{|l|}{14} & \multicolumn{2}{|l|}{9} & \multicolumn{2}{|l|}{6} \\
\hline
\end{tabular}

Table- 3 shows regional distribution of lymphadenopathy. In this study it was observed that the most common site of LAP was Cervical (45.3\%). Followed by Supraclavicular(22.6\%), Axillary(18.4\%) and submental(7.5\%). Post. Auricular(5.0\%) and inguinal $(0.08 \%)$ were found to be least common sites.

Table -3 : Regional distribution of lymphadenopthy

\begin{tabular}{|l|l|l|}
\hline Group of Lymph node involved & No of cases & $\%$ \\
\hline Cervical & 54 & $45.3 \%$ \\
\hline Submental & 9 & $7.5 \%$ \\
\hline Post. Auricular & 6 & $5.0 \%$ \\
\hline Supraclavicular & 27 & $22.6 \%$ \\
\hline Axillary & 22 & $18.4 \%$ \\
\hline Inguinal & 1 & $0.08 \%$ \\
\hline & 119 & $100 \%$ \\
\hline
\end{tabular}

Table- 4 shows regional distribution of LAP compared with their cytological classification. It was observed that Tuberculous lesions most frequently effected the cervical lymph nodes (48.4\%). Malignant conditions showed frequent involvement of supraclavicular nodes (see Table 4). In some cases where more than one lymphnode group was involved, category was decided by the most prominent involvement. Cervical lymphadenopathy was however most common presentation due to any of the reasons.

Table 4: Peripheral lymphnode groups involved in various lymphadenopathy

\begin{tabular}{|l|l|l|l|l|l|l|l|l|l|l|}
\hline \multirow{2}{*}{ Lymph Node group } & Tuberculous & \multicolumn{2}{l|}{ Reactive } & \multicolumn{2}{l|}{ Pyogenic } & \multicolumn{2}{l|}{ Lymphoma } & \multicolumn{2}{l|}{ Metastasis } \\
\cline { 2 - 13 } & No & $\%$ & No & $\%$ & No & $\%$ & No & $\%$ & No & $\%$ \\
\hline Cervical & 30 & $48.4 \%$ & 10 & $40.0 \%$ & 8 & $36.36 \%$ & 3 & $75.00 \%$ & 3 & $50.00 \%$ \\
\hline Submental & 2 & $3.2 \%$ & 3 & $12.0 \%$ & 4 & $18.18 \%$ & 0 & $00 \%$ & 0 & $00 \%$ \\
\hline Post. auricular & 4 & $6.4 \%$ & 2 & $8.0 \%$ & 0 & $00 \%$ & 0 & $00 \%$ & 0 & $00 \%$ \\
\hline supraclavicular & 15 & $24.9 \%$ & 5 & $20.0 \%$ & 4 & $18.18 \%$ & 1 & $25.00 \%$ & 2 & $33.33 \%$ \\
\hline Axillary & 10 & $16.1 \%$ & 5 & $20.0 \%$ & 6 & $27.27 \%$ & 0 & $00 \%$ & 1 & $16.66 \%$ \\
\hline Inguinal & 1 & $1.6 \%$ & 00 & $00 \%$ & 0 & $00 \%$ & 0 & $00 \%$ & 0 & $00 \%$ \\
\hline & 62 & & 25 & & 22 & & 4 & & 6 & \\
\hline
\end{tabular}

Table - 5 shows Chest X-ray findings of cytologically classified cases of peripheral lymphadenopathy. Metastatic lymphadenitis though less common in itself but frequently shows radiological changes like pleural effusion $(33.3 \%)$, lung parenchyma $(33.3 \%)$ and mediastinal involvement $(33.3 \%)$. Lymphomas too showed mediastinal involvement and pleural effusion. There was no significant radiological finding $(0 \%)$ in pyogenic and reactive lymphadenitis. In tuberculous lesions, Lung parenchyma was most frequently involved.

Table- 5: Incidence of different chest X- ray abnormality detected in various pathological type of lymphadenopathy

\begin{tabular}{|l|l|l|l|l|l|l|l|}
\hline Pathological variety (No) & \multicolumn{2}{l|}{$\begin{array}{l}\text { Mediastinal } \\
\text { involvement }\end{array}$} & \multicolumn{2}{l|}{ Lung parenchyma involvement } & \multicolumn{2}{l}{ Pleural effusion } \\
\cline { 2 - 9 } & No & $\%$ & No & $\%$ & No & $\%$ \\
\hline Tuberculous lympadinitis (62) & 4 & $06.45 \%$ & 22 & $35.48 \%$ & 8 & $12.90 \%$ \\
\hline Reactive lymphadenitis (25) & 0 & $00 \%$ & 0 & $00 \%$ & 0 & $00 \%$ \\
\hline Pyogenic Lymphadinitis (22) & 0 & $00 \%$ & 0 & $00 \%$ & 0 & $00 \%$ \\
\hline Lymphoma $\quad(4)$ & 2 & $50.00 \%$ & 0 & $00 \%$ & 2 & $50.00 \%$ \\
\hline Metastatic lymphadenopathy(6) & 2 & $33.33 \%$ & 2 & $33.33 \%$ & 2 & $33.33 \%$ \\
\hline
\end{tabular}




\section{Discussion}

Peripheral lymphadenopathy (LAP) is a frequent finding caused due to a local or systemic, benign, self-limited, infectious condition. It can also be a manifestation of underlying malignancy like lymphoma or metastasis from other sites. The most common etiology can vary from place to place depending upon the incidence and prevalence of main underlying pathological condition in different geographical areas. In many parts of India tuberculosis is the main non-malignant cause of LAP in adults and children. The observed incidence may sometimes also vary depending on the department which is studying the cases and collecting the data. Jharkhand state still has a high prevalence of $\mathrm{TB}$ in addition to other common pathologies. ${ }^{7}$ Enlarged/palpable peripheral lymph nodes is a common presenting complaint in Department of T.B. \& Chest, Rajendra Institute of Medical Sciences (RIMS), Ranchi. Out of the total of 119 patients with peripheral lymphadenopathy included in this study it was observed that the frequency was higher in males in all age groups(Figure-1)which was similar to findings of some other regional studies in India. ${ }^{6,8}$ Overall highest number of cases were observed in 21-30 yr age group. Other studies in india have shown similar results. Some studies on lymphadenitis observed a second peak in 51-60 yr age group. ${ }^{11}$

FNAC findings showed that Tuberculous lymphadenitis was overall the most common etiology $(52 \%)($ Table-1). This observation most likely may be due to a higher prevalence of TB in the geographical area of the study population. ${ }^{7}$ Reports of other similar studies conducted in northern nigeria, jammu and Bangalore have shown similar finding. ${ }^{\mathbf{3 , 5}, \mathbf{6}}$ However two recent studies conducted by Garg et al in patiala and Chawla et al from Dehradun reported a higher prevalence of reactive lyphadenitis in their study population. ${ }^{\mathbf{8}, 12}$ In younger age group(10-20 yrs) beningn reactive lymphadenitis was more common(Table-2). There were fewer cases showing malignant etiologies but were relatively more frequently observed in older age groups, similar to observations of Chawla et al. ${ }^{8}$

We observed that cervical lymph nodes were the most common group of lymphnode involved(Table-3) as also observed by Garg et al and Khajuria et al. ${ }^{6,12}$ The likely cause is that TB which has a high prevalence in the study population, most often involves the cervical lymph nodes (Table-4) confirmed by other reports. ${ }^{\mathbf{1 , 1 1}, 13}$ Supraclavicular lymphadenitis was found to be most frequently associated with malignant conditions like lymphoma and metastasis. Several other authors have confirmed this finding. ${ }^{\mathbf{1 , 2}}$

We observed that changes Chest X-ray were more commonly observed in malignant changes and Tuberculous lesions. Metastatic lymphadenitis frequently shows radiological changes like pleural effusion (33.3\%), lung parenchyma (33.3\%) and mediastinal involvement (33.3\%). Lymphomas too showed mediastinal involvement and pleural effusion. There was no significant radiological finding $(0 \%)$ in beningn conditions like pyogenic and reactive lymphadenitis. In tuberculous lesions, Lung parenchyma was most frequently involved. Our findings were in agreement with in one study by Shah et al in Dhaka. ${ }^{14}$ There was a satisfactory correlation between cytological and radiological findings.

\section{Conclusion}

Peripheral Lymphadenopathy is a common clinical presentation in several local or systemic disorders. In the current observational study it was found that the majority of patients presenting with peripheral lymphadenopathy were diagnosed having tuberculous lesions most likely due to a high prevalence of TB in Ranchi zone. FNAC is a simple, safe procedure and can be accepted as a reliable technique to establish a cytological diagnosis in lymphadenopathies. Cervical group of lymph nodes are most prone to be effected. In addition, lymphomas and metastasis frequently involve the supraclavicular lymph nodes. Chest X-ray abnormalities are more common in tuberculous and Malignant causes of LAP. Further procedure for histopathological study may be required in some cases associated with multiple other risk factors.

\section{References}

[1]. Mohseni S, Shojaiefard A, Khorgami Z, Alinejad S, Ghorbani A, Ghafouri A . Iran J Med Sci Supplement March 2014; 39(2):158-70

[2]. Bazemore AW, Smucker DR. Lymphadenopathy and malignancy. Am Fam Physician. 2002 Dec 1;66(11):2103-10.

[3]. Ochicha O, Edino ST, Mohammed AZ, Umar AB, Atanda AT. Pathology of peripheral lymph node biopsies in Kane, Northern Nigeria. Ann Afr Med. 2007 Sep;6(3):104-8

[4]. A.K. Chakraborty. Epidemiology of tuberculosis : Current status in India. Indian J Med Res 120, October 2004: 248-76

[5]. Sreenidhi G.M, Nandeeshkumar G.N, Clinicopathological study of Cervical Tubercular Lymphadenopathy at KIMS Hospital Bangalore. Journal of Evolution of Medical and Dental Sciences Nov 2013; 2(44):8655-66

[6]. Khajuria R, Goswami KC, Singh K, Dubey VK. Pattern of Lymphadenopathy on Fine Needle Aspiration Cytology in Jammu. JK Science. Jul-Sep 2006; 8(3):157-59

[7]. TB INDIA 2016. Revised National TB Control Program, Annual Status Report. 2016:41-44

[8]. Chawla N, Kishore S, Kudesia S. FNAC of Lymph Node Disorders. Indian Medical Gazette. Aug 2012: 312-15

[9]. Choudhary RK, Singh PK, Kumar D. Clinicopathological Study of Cervical Tubercular Lymphadenopathy at Rajendra Institute of Medical Sciences, Ranchi. IOSR Journal of Dental and Medical Sciences.Jan 2017;16(1):12-15

[10]. Kasana D , Verma J, Dhavan I, Prasad HK. Comparative Analysis of Various Diagnostic Techniques for Tubercular Lymphadenitis: A Pilot Study from a Resource Poor Country. JIMSA July-Sep 2012; 25(3): 167-69 
[11]. Memish ZA, Mah MW, Mahmood SA, Bannatyne RM, Khan MY. Clinico-diagnostic experience with tuberculous lymphadenitis in Saudi Arabia. Clin Microbiol Infect 2006: 137-41

[12]. Garg P, Kundal R, Mittal S, Singh K. Clinicopathological Correlation of Lymphadenopathy. ejbps, 2016; 3(8):305-8

[13]. Kochhar AK, Patel KB. , Shah M. Pattern of Lymphadenopathy on Fine Needle Aspiration Cytology of Superficial Lymph Nodes. Journal of Advance Researches in Biological Sciences, 2012; 4(4): 288-92

[14]. Shah R, Talukdar DC, Fakir MAY, Hossain MZ, Khan AFM, Shaha PS, Kashem MA. Role of Chest X-Ray in Tuberculous Cervical Lymphadenitis. J Dhaka Med Coll. 2013; 22(2): 125-28 